\title{
Diseño de programas de asignaturas basados en competencias y su aplicación en la Universidad del Bío-Bío, Chile
}

\author{
Design of course programs based on competences and their application \\ at the Universidad del Bío-Bío, Chile
}

Fabricio Salgado $^{1} \quad$ José Corrales $^{1} \quad$ Luis Muñoz $^{1} \quad$ Juan Delgado $^{1}$

Recibido 5 de abril de 2011, aceptado 16 de mayo de 2012

Received: April 5, 2011 Accepted: May 16, 2012

\begin{abstract}
RESUMEN
La Universidad del Bío-Bío está en proceso de implantación de un modelo educativo basado en competencias. En este contexto, los programas de asignaturas deben migrar para adecuarse a este modelo educativo. Este trabajo muestra que el cambio del modelo educativo se refleja en los programas de asignatura proveyendo en forma consistente y coherente la información necesaria y clara sobre lo que el alumno aprenderá: competencias o aprendizajes esperados, las estrategias formativas que se utilizarán, las actividades que se realizarán y la forma en que se le evaluará: estrategias, criterios e indicadores. Se indican algunos aspectos a considerar mostrando un ejemplo de aplicación, constituyéndose en uno de los primeros esfuerzos en la implementación del modelo educativo institucional dentro del departamento de Ingeniería Eléctrica y Electrónica de la Universidad del Bío-Bío.
\end{abstract}

Palabras clave: Diseño, competencias, programas de asignaturas, modelo educativo, estrategias formativas.

\begin{abstract}
The Universidad del Bío-Bio is in process of establishment of an educational model based on competences. In this context, the course programs should be adapted to the new educational model. This paper shows that the change of the educational model is manifested in course programs providing consistent, coherent and clearly the necessary information that the student will learn: competences or expected learning, formative strategies that will be used, activities that are carried out, and the way the students will be evaluated: strategies, criteria and indicators. Some guides are given for the design of a course program and an application example is shown. This work is one of the first efforts in the establishment of the institutional educational model at the Department of Electric and Electronic Engineering at the Universidad del Bío-Bío.
\end{abstract}

Keywords: Design, competences, course programs, educative model, formative strategies.

\section{INTRODUCCIÓN}

Las experiencias internacionales han sido un referente para la aplicación de la metodología de formación basada en competencias, esto es, países de la organización económica de cooperación para el desarrollo: Reino Unido, Francia, Canadá, España,
Australia tienen sistemas de certificación en base a competencias. En países de América Latina, como: México, Brasil, Argentina, Costa Rica y Chile se están iniciando procesos de certificación de competencias para el medio laboral y sistemas de formación basados en competencias. Desde el punto de vista del trabajo, la OIT (Organización

\footnotetext{
1 Depto. de Ingeniería Eléctrica y Electrónica. Facultad de Ingeniería. Universidad del Bío-Bío. Av. Collao 1202. Concepción, Chile.E-mail: fsalgado@ubiobio.cl; jcorrale@ubiobio.cl; 1munozs@ubiobio.cl; jdelgado@ubiobio.cl
} 
Internacional del Trabajo) [1], mediante el Centro Interamericano de Investigación y Documentación sobre la Formación Profesional (CINTERFOR) [2], está apoyando iniciativas en la materia. Lo mismo sucede con la OPS/OMS (Organización Panamericana de la Salud/Organización Mundial de la Salud) [3] en conjunto con CINTERFOR; por otro lado, el sistema financiero como el Banco Interamericano de Desarrollo y el Banco Mundial, se encuentra financiando proyectos en Argentina, Brasil, Chile.

En respuesta a esto, la Universidad del Bío-Bío se ha replanteado la misión, visión y valores compartidos, generando un modelo educativo y dando como resultado programas de pregrado, en función de sus propias necesidades, formalizando un perfil adecuado para sus egresados en consideración de su propio sello universitario.

La educación en base a competencias surge de la necesidad de disminuir la brecha existente entre las competencias de un egresado, respecto a las que requiere un mercado competitivo y cambiante. $\mathrm{El}$ modelo de enseñanza además recoge el concepto de movilidad del estudiante, por lo cual las competencias deben ser normalizadas y estandarizadas, esto a nivel nacional y en un futuro a nivel internacional, a través del Sistema de Créditos Transferibles (SCT). La calidad de la educación hoy en día se mueve hacia el concepto de empleabilidad. Bajo éste, el objetivo de la educación universitaria se orienta en dar las herramientas necesarias para que sus egresados tengan las mayores posibilidades para insertarse en el mundo laboral, tal como se menciona en [4].

\section{CONSIDERACIONES CONCEPTUALES}

\section{El concepto de competencia}

La primera referencia del uso de la palabra Competencia en el ámbito de la educación corresponde a Noam Chomsky [5]. Las teorías de Chomsky acerca de la adquisición del lenguaje están basadas en el concepto de competencia y performance, entendiéndose competencia como la capacidad genética de la adquisición del lenguaje de acuerdo a reglas gramáticas, mientras que performance es entendida como el acto de comunicarse con el lenguaje. Hoy en día, la definición de competencia abarca más características, de las que se refiere
Chomsky, existiendo variadas definiciones. Por otro lado, es importante recordar que las competencias requeridas deben derivar de los objetivos que se haya propuesto cumplir una organización. Es por tanto necesario que una organización tenga un concepto propio y oficial en este término.

Particularmente, el tipo de competencia que interesa en el área de la educación se refiere a las competencias profesionales. El modelo educativo planteado en la Universidad del Bío-Bío las define como: La capacidad que posee una persona que le permite aplicar un conocimiento a través de una habilidad (cognitiva, psicomotora, social, afectiva) en un contexto laboral/profesional específico, respondiendo a estándares de calidad aceptados para el ámbito en que se circunscribe. En esta definición se encuentra una asociación entre habilidades que se entienden como procesos cognitivos genéricos, y un modelo que agrupa conocimiento. Particularmente son cuatro los dominios o áreas temáticas sobre las cuales se pueden agrupar las competencias deseables y que son definidas como saberes: saber, saber hacer, saber ser y saber convivir. Estas cuatro combinaciones de saberes fueron propuestas por la Comisión Internacional sobre Educación Superior para el siglo XXI a la UNESCO y provienen de la aplicación de conocimiento (saber), habilidades (hacer) y actitudes (ser y convivir). En particular el "convivir" y el "ser" no se plantean en este trabajo como saberes separados, sino como uno en el "saber ser".

En las definiciones dadas por competencia por la OIT, la Asociación Francesa de Normalización (AFNOR) [6], CINTERFOR y el proyecto Tunning [7], se puede notar que existe una triple cualidad de ser competente: saber, saber hacer y saber ser, que identifica el concepto de competencia para todas. $\mathrm{Su}$ consideración está relacionada con el surgimiento de lo que se conoce como "pensamiento complejo". Esto es, se considera toda parte en el proceso de adquisición del conocimiento como importante, si bien es cierto, en la enseñanza siempre se fue partidario de limitar ésta a un proceso de adquisición basado en habilidades cognitivas, desechando otras, hoy en día se tiene el consenso que este tipo de enseñanza hace que se pierdan ciertos conocimientos que podrían ser importantes, según el enfoque del pensamiento complejo. 


\section{La clasificación de las competencias}

La misma triple cualidad de las competencias, del saber, saber hacer y saber ser, proporciona una forma de clasificación. Es así que el modelo Tunning las separa en competencias genéricas y específicas. Las competencias genéricas pueden ser definidas como las habilidades necesarias para el empleo y la vida como ciudadano, importantes para todos, cualquiera sea la carrera que se curse; desde este punto de vista son transversales a diferentes ámbitos de la actividad personal y laboral. Estas pueden ser divididas en:

Competencias instrumentales: competencias que tienen una función instrumental, entre ellas se encuentran las habilidades cognitivas, la capacidad de comprender y manipular ideas y pensamientos. Las capacidades metodológicas para manipular el ambiente, ser capaz de organizar el tiempo y las estrategias para el aprendizaje, tomar decisiones o resolver problemas. Destrezas tecnológicas relacionadas con el uso de maquinaria, destrezas de computación y gestión de la información. Destrezas lingüísticas tales como la comunicación oral y escrita o conocimiento de una segunda lengua.

Competencias interpersonales: capacidades individuales relativas a la capacidad de expresar los propios sentimientos, habilidades críticas y de autocrítica. Destrezas sociales asociadas con las habilidades interpersonales, la capacidad de trabajar en equipo o la de expresión de compromiso social o ético. Estas competencias tienden a facilitar los procesos de interacción social y cooperación.

Competencias sistémicas: son las destrezas y habilidades que conciernen a los sistemas como totalidad. Suponen una combinación de la comprensión, la sensibilidad y el conocimiento que permiten al individuo ver cómo las partes de un todo se relacionan y se agrupan. Estas capacidades incluyen la habilidad de planificar los cambios de manera que puedan hacerse mejoras en los sistemas como un todo y diseñar nuevos sistemas. Las competencias sistémicas o integradoras requieren como base la adquisición previa de competencias instrumentales e interpersonales.

Otras separaciones pueden ser hechas tal como lo plantea el modelo educativo de la Universidad del Bío-Bío en base a la triple cualidad que se les ha dado a las competencias, existiendo una relación entre algunos términos que se usarán a lo largo de este trabajo; por supuesto esta relación no es absoluta, pero será útil, ya que se usa indistintamente en algunos textos. La Figura 1 resume la relación mencionada.

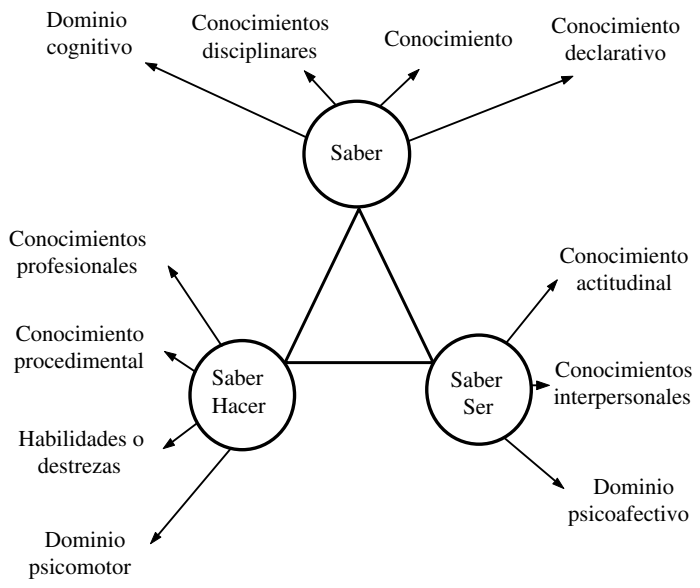

Figura 1. Relación entre algunos términos y los diferentes saberes.

Respecto a lo anterior, es posible decir que un término que alude los tres saberes es la palabra "conocimiento", a pesar de que en algunos casos sólo se reserva para ser usada cuando se habla del "saber". Aún así, según el contexto en que se utilice se asume conocido a cuál de los dos significados se refiere. Lo mismo sucede con las palabras: capacidad, habilidades y destrezas, utilizadas en algunos casos como sinónimos de la palabra competencia.

\section{Taxonomías y nivel de dominio de las competencias}

Las competencias que se esperan de un determinado profesional pueden ser agrupadas en dominios o áreas de aprendizaje, las cuales deben cubrirse en los diversos niveles de la enseñanza, de forma de garantizar una formación integral. El nivel de logro de las competencias puede evaluarse adoptando ciertas taxonomías para cada dominio, tanto para el dominio del conocimiento, las habilidades y las actitudes.

Una taxonomía es un sistema de clasificación y ordenamiento de los objetivos pedagógicos en función de su ámbito y complejidad, éstas miden el desarrollo del conocimiento, de un afecto o de una actitud. Particularmente para el nivel de logro relacionado con el conocimiento, se ha adoptado 
universalmente el uso de la taxonomía de Bloom [8], la cual es práctica y entendible dando énfasis a la redacción de objetivos y sus actividades desde el punto de vista cognitivo. Sin embargo, este mismo hecho no considera que la enseñanza-aprendizaje abarca más que sólo el pensamiento, sino los sentimientos, creencias y el ambiente sociocultural de enseñanza, entre otras cosas. Una de las más importantes premisas de esta taxonomía es que cada categoría o nivel del conocimiento debe ser dominada antes de continuar con la siguiente y cada nivel es superior en dificultad al otro (algo discutido hoy en día), siendo sus niveles los siguientes: Conocimiento, Comprensión, Aplicación, Análisis, Síntesis y Evaluación. La taxonomía de Bloom ha sido estudiada por varios autores como Anderson y Krathwohl [9], quienes ajustaron el modelo de Bloom invirtiendo los niveles de síntesis y evaluación y agregando dimensiones del conocimiento (factual, conceptual, procedimental y metacognitivo). Bloom también desarrolló una taxonomía en el dominio afectivo [10] proponiendo los niveles de: Recepción, Respuesta, Valoración, Conceptualización e Internalización, existiendo sutiles diferencias entre los tres últimos, haciendo poco clara la manera en que son separados dentro de la taxonomía. Se debe mencionar además que Bloom originalmente identificó un tercer dominio (psicomotor), pero éste no fue detallado por completo dando cabida a otras taxonomías como la de Dave [11] cuyo modelo es sencillo y fácil de aplicar en ambientes de desarrollo colectivo. Dave propone para dicho dominio los niveles de: Imitación, Manipulación, Precisión, Articulación y Naturalización. Otras taxonomías sugeridas para el dominio psicomotor son las de Dawson [12], Simpson [13] y Harrow [14]. Particularmente Harrow interpreta el dominio psicomotor predispuesto fuertemente hacia el desarrollo de la forma física, destrezas y agilidad además del control del cuerpo, por lo que se hace más apropiado para el desarrollo de movimientos corporales en niños. Simpson, por otro lado, difiere de la taxonomía de Dave, ya que propone dos niveles previos al nivel de Imitación (Percepción y Preparación), siendo particularmente útil su aplicación en adultos en ambientes que no les son confortables como situaciones de conflicto y rutinas en situaciones de emergencia. En este dominio los autores Ferris y Aziz [15] propusieron una taxonomía enfocada al campo de la educación universitaria, interpretando el concepto de dominio psicomotor como el desarrollo de las habilidades o destrezas asociadas con el desempeño en una profesión. Particularmente esta taxonomía es útil para el desarrollo del trabajo de laboratorio en programas de estudio de ingenieros, planteando los niveles de: Reconocimiento de herramientas y materiales, Manipulación de herramientas y materiales, Operación básica de herramientas, Operación competente de herramientas, Operación experta de herramientas, Planificación de operaciones de trabajo y Evaluación de resultados y Planificación por medio de la mejora.

Otro autor Frabboni [16] realiza una integración de diferentes escalas taxonómicas entre las que se encuentra Bloom, separando su taxonomía en tres niveles: uno de aprendizajes elementales (centrados en el saber), de aprendizajes medios (centrados en el comprender) y superiores (descomponer e integrar).

Una taxonomía ayudará a definir el nivel de logro, en los tres dominios, en que se agrupan las competencias que se quiere implantar, este nivel de logro será de menor a mayor desarrollo.

\section{Programas de asignaturas en perspectivas de competencias}

Una asignatura puede o no actuar sola para implantar competencias en los estudiantes [17]. Puede formar parte de un conjunto de asignaturas que refuerzan ciertas competencias, así como también puede actuar implantando nuevas competencias a los estudiantes. Los pasos a seguir para su confección son los siguientes:

$\square$ Identificación de las competencias derivadas del diagnóstico y plan formativo deseable. En este punto es necesario reconocer fines y principios institucionales, reconocer características (en términos de competencias) del perfil del egresado de la carrera en la cual se inscribe la asignatura. Conocer el grado de acercamiento que poseen los estudiantes a las competencias elaboradas (diagnóstico). Reconocer los aportes que la asignatura hace al perfil del egresado (en términos de competencias) en consenso con las asignaturas de la misma área y del mismo año en que se dicta.

$\square$ Desplegar los aprendizajes esperados en las tres dimensiones: conceptual, procedimental y actitudinal. 
$\square$ Identificar la/las estrategias formativas.

$\square$ Seleccionar y organizar las actividades en orden progresivo y secuencial.

$\square$ Seleccionar las estrategias de evaluación.

$\square$ Identificar los criterios e indicadores de evaluación según nivel de dominio. En este punto resulta importante elaborar evidencias para verificar el grado de desarrollo de las competencias que nos hemos propuesto desarrollar (indicadores de logro). Valorar la intervención didáctica en relación a los indicadores definidos. Trazar un plan de intervención didáctica e implementarlo.

$\square$ Señalar la estrategia de retroalimentación.

\section{Asignación de créditos bajo un modelo basado en competencias}

El modelo de educación basado en competencias considera el trabajo realizado por el estudiante en su totalidad, tanto dentro como fuera del aula, por lo tanto, los créditos que se le asignan a una asignatura deben reflejar las horas de trabajo que dedica un estudiante para cumplir con los requisitos de la misma. Algunos principios para la asignación de créditos en el caso del SCT chileno [18], son:

च 60 créditos académicos representan el tiempo de dedicación para que un estudiante a tiempo completo logre los resultados de aprendizaje de un año de estudios. Este tiempo varía entre $1.440 \mathrm{hrs} / a$ ano y $1.900 \mathrm{hrs} / \mathrm{año}$.

$\square$ La asignación de créditos a una actividad curricular se basa en la carga de trabajo del estudiante.

$\square$ Cada actividad curricular tiene asignado un número de créditos como proporción del total anual, el que se expresa en enteros.

ఐ El trabajo total del estudiante sólo puede ser medido por aproximación, puesto que el universo estudiantil presenta alta dispersión y los métodos para recoger información tienen baja confiabilidad.

ஏ Una actividad curricular, no importando el plan de estudios en que se encuentre, tiene un único valor en créditos al interior de una institución.

$\square$ La obtención de créditos por parte de un estudiante supone una evaluación y el haber superado los mínimos establecidos.

Respecto a lo anterior es posible encontrar los métodos para asignar créditos en [19].
Particularmente la Universidad del Bío-Bío determina el valor del crédito SCT en función de: 50 horas de trabajo semanal que el estudiante emplea en sus actividades académicas durante 36 semanas lectivas anuales, lo que resulta en 1.800 horas de dedicación. Si el valor anterior se normaliza a 60 créditos, resulta que un crédito SCT equivale a 30 horas de dedicación.

\section{La evaluación}

La evaluación basada en competencias debe ser integral, esto es, combinar conocimiento, entendimiento, solución de problemas, habilidades técnicas, actitudes y ética. La evaluación debe estar orientada al problema, ser interdisciplinaria, cubrir grupos de competencias, exigir habilidades analíticas, combinar la teoría con la práctica.

En la evaluación basada en competencias, los profesores emiten juicios fundamentados en una variedad de evidencias que demuestran hasta dónde un estudiante satisface los requisitos exigidos por un estándar o conjunto de criterios. Por otro lado, no sólo se utiliza para certificar ciertas competencias adquiridas por el estudiante sino también para ayudar en el proceso formativo, por lo que el término evaluación no debe ser ligado aquí a una nota o calificación.

Para ello el profesor debe:

$\square$ Establecer los criterios de evaluación (logros, e indicadores de logro), los cuales deben ser familiares a los estudiantes para que ellos puedan juzgar hasta dónde dichos criterios han sido satisfechos).

$\square$ Decidir la evaluación con base en la comparación entre los logros esperados y las evidencias detectadas.

$\square \quad$ Registrar los resultados.

$\square$ Revisar los procedimientos utilizados en la evaluación.

Los tipos de evaluación según [20] pueden ser categorizados de acuerdo a: la intención que se tenga para llevarla a cabo, al momento en que se aplique, según la extensión, según el rol del evaluador, según un estándar de comparación o un referente de contrastación. A continuación se indican los tipos de evaluación según esta clasificación mencionada. 
Según la intencionalidad:

$\square$ Formativa, la que permite emitir un juicio sobre el desempeño del estudiante con la finalidad de ayudarlo a aprobar el curso, generalmente no se califica. Dentro de esta se encuentra la evaluación diagnóstica, por medio de la cual se establece el nivel de conocimientos del estudiante en una cierta materia. Permite identificar las dificultades y debilidades en una materia dada.

$\square$ Sumativa, se califica la suma de los conocimientos adquiridos, esto es, se emite un juicio sobre el desempeño del estudiante en función de ciertos criterios, de forma de ser promovido de curso o a un nivel superior.

Según el momento en que se realizan:

घ Evaluación inicial, de proceso, final y parcial.

Según la extensión:

Si se desea evaluar los objetivos de aprendizaje, la evaluación puede ser:

ఐ Global. Considera la totalidad de los objetivos de aprendizaje.

ఐ Parcial. Considera sólo una parte de los objetivos de aprendizaje.

Según el rol de evaluador:

$\square$ Interna. Preparadas y aplicadas por personas como el docente.

$\square$ Autoevaluación. El estudiante evalúa sus aprendizajes.

$\square$ Heteroevaluación. La utilizada con mayor frecuencia, en donde el docente evalúa y el estudiante solamente responde las preguntas.

$\square$ Coevaluación. Evaluación de tipo colectiva, todos los estudiantes de un grupo participan del proceso evaluativo.

$\square$ Externa. Llevadas a cabo por personas ajenas, ya sean del proceso educativo o de la institución.

Según un referente de contrastación:

$\square$ Normativa. La evaluación de un alumno se basa en una medida de tendencia central, por ejemplo, el promedio aritmético, por lo que existe un grupo de comparación en base al cual se definen estándares o normas para comparar los resultados obtenidos.

$\square$ Referida a criterio. Al contrario del anterior, la referencia de comparación con otros alumnos no existe y se evalúa en función de un criterio preexistente.

Evidencias para la evaluación de competencias Las competencias no son observables, por lo que hay que inferirlas por medio de evidencias, que no son otra cosa que elementos tangibles que permiten demostrar que se ha logrado cubrir de manera satisfactoria: un requerimiento, un criterio específico de desempeño, una competencia o bien el resultado de un aprendizaje. Las evidencias pueden ser separadas en dos tipos: de conocimiento y de desempeño, estas últimas a su vez se dividen en de desempeño propiamente tal y de producto.

Las evidencias de conocimiento se relacionan con la construcción del conocimiento, teorías, principios, todo aquello que represente un punto de partida para la construcción de aprendizajes posteriores. Conocimientos que fundamentan la práctica.

Las evidencias de desempeño directo aluden al comportamiento en sí mismo en situaciones de simulación o reales. Cuando se desea expresar las evidencias esperadas de un estudiante de forma de compararlas con el logro de los objetivos planteados, la redacción a realizar tiene una estructura en que se anteponen las acciones a demostrarse en una secuencia determinada, tal como se indica a continuación:

$$
\text { Acción + Objeto + Condición }
$$

Por ejemplo:

Verifica las condiciones del área de trabajo. Limpia el equipo según las especificaciones.

Las evidencias de producto corresponden a la recopilación de signos concretos que pueden visualizarse $o$ analizarse en el producto mediante diversos tipos de ensayos. Al igual que las evidencias de desempeño directo, su estructura es como sigue:

$$
\text { Objeto + Acción + Condición }
$$

Por ejemplo:

El medicamento fue administrado siguiendo el procedimiento establecido. 


\section{Técnicas e instrumentos de evaluación}

Las técnicas de evaluación son los métodos que se utilizan para recoger evidencias de conocimiento y de desempeño, entre estas se encuentran las técnicas de observación directa, análisis de realizaciones, técnicas de información directa y las pruebas-cuestionarios. Las técnicas de evaluación que se pueden utilizar son variadas y se aplican mediante un instrumento de evaluación con un cierto motivo. El uso de algunas técnicas e instrumentos de acuerdo al tipo de evidencia que se desea recoger puede resumirse en la Tabla 1. La Tabla 2 presenta una descripción de éstos de forma de facilitar su elección.

Tabla 1. Técnicas e instrumentos de evaluación.

\begin{tabular}{|c|c|c|c|c|}
\hline Nombre & Descripción & Para qué & Cómo & Observaciones \\
\hline Examen oral & $\begin{array}{l}\text { Método imprescindible } \\
\text { para medir los } \\
\text { objetivos educacionales } \\
\text { que tienen que ver con } \\
\text { la expresión oral. }\end{array}$ & $\begin{array}{l}\text { Para comprobar la } \\
\text { profundidad en la } \\
\text { comprensión, la } \\
\text { capacidad de relacionar } \\
\text { diversas materias y } \\
\text { el conocimiento de } \\
\text { problemas actuales, } \\
\text { temas conflictivos. }\end{array}$ & $\begin{array}{l}\text { Definir con claridad el } \\
\text { objetivo del examen } \\
\text { y lo que se va a tener } \\
\text { en cuenta, así como } \\
\text { estructurar algún } \\
\text { procedimiento: escalas } \\
\text { y guías de observación. }\end{array}$ & $\begin{array}{l}\text { Se puede instrumentar } \\
\text { de forma variada: } \\
\text { defensa de un } \\
\text { proyecto, entrevista } \\
\text { con el profesor, } \\
\text { presentaciones } \\
\text { grupales, debates entre } \\
\text { alumnos, ponencias. }\end{array}$ \\
\hline $\begin{array}{c}\text { Prueba escrita } \\
\text { de respuesta } \\
\text { abierta }\end{array}$ & $\begin{array}{l}\text { Prueba cronometrada } \\
\text { efectuada bajo control } \\
\text { en la que el alumno } \\
\text { construye su respuesta. }\end{array}$ & $\begin{array}{c}\text { Para comprobar } \\
\text { la capacidad de } \\
\text { expresión, la } \\
\text { organización de } \\
\text { ideas, la capacidad de } \\
\text { aplicación, el análisis y } \\
\text { la creatividad. }\end{array}$ & $\begin{array}{c}\text { Tras redactar las } \\
\text { preguntas y dada la } \\
\text { difícil objetividad, } \\
\text { llegado el momento } \\
\text { de la corrección, es } \\
\text { importante tener claro } \\
\text { los criterios y los } \\
\text { diferentes grados de } \\
\text { realización. } \\
\end{array}$ & $\begin{array}{c}\text { Admiten varias } \\
\text { modalidades: una } \\
\text { pregunta de respuesta } \\
\text { amplia, varias } \\
\text { preguntas de respuesta } \\
\text { breve en torno a un } \\
\text { tema y exámenes de } \\
\text { libro abierto. }\end{array}$ \\
\hline $\begin{array}{l}\text { Pruebas } \\
\text { objetivas } \\
\text { (tipo test) }\end{array}$ & $\begin{array}{c}\text { Examen escrito } \\
\text { estructurado con } \\
\text { diversas preguntas, en } \\
\text { las que el alumno no } \\
\text { elabora una respuesta, } \\
\text { sólo habrá de señalarla } \\
\text { o completarla con } \\
\text { elementos muy } \\
\text { precisos. }\end{array}$ & $\begin{array}{c}\text { Permiten evaluar } \\
\text { sobre una base amplia } \\
\text { de conocimientos y } \\
\text { diferenciar bien el } \\
\text { nivel de adquisición } \\
\text { del conocimiento de } \\
\text { los alumnos. }\end{array}$ & $\begin{array}{l}\text { Se debe determinar } \\
\text { qué se debe preguntar } \\
\text { y cómo hacerlo, } \\
\text { para luego generar } \\
\text { preguntas sobre algo } \\
\text { que sea importante } \\
\text { saber. El conjunto debe } \\
\text { resultar equilibrado. }\end{array}$ & $\begin{array}{l}\text { Las opiniones de } \\
\text { respuesta deben tener } \\
\text { una longitud similar } \\
\text { y una conexión } \\
\text { con la pregunta. } \\
\text { Además, deben ser } \\
\text { del mismo ámbito y } \\
\text { no ser sinónimas ni } \\
\text { sin sentido y debe } \\
\text { haber una claramente } \\
\text { correcta. }\end{array}$ \\
\hline
\end{tabular}

Tabla 2. Técnicas e instrumentos para la evidencia del conocimiento.

\begin{tabular}{|c|c|c|c|}
\hline Técnica e instrumento & Conocimiento declarativo & Destrezas/habilidades & Actitudes \\
\hline Prueba objetiva & Recomendado & - & - \\
\hline Resolución de problemas & Recomendado & Recomendado & Recomendado \\
\hline Mapa conceptual & Recomendado & Recomendado & - \\
\hline Examen oral & Recomendado & - & Recomendado \\
\hline Diario & - & Recomendado & Recomendado \\
\hline Observación & - & Recomendado & Recomendado \\
\hline Debate & - & Recomendado & Recomendado \\
\hline Caso & Recomendado & Recomendado & Recomendado \\
\hline Proyecto & Recomendado & Recomendado & Recomendado \\
\hline Portafolio & Recomendado & Recomendado & Recomendado \\
\hline Ensayo & Recomendado & & - \\
\hline
\end{tabular}


Las técnicas auxiliares más utilizadas para ayudar a recoger las evidencias de desempeño son el uso de las rúbricas o matriz de valoración, las escalas y las listas de cotejo. Las rúbricas son una guía para describir los criterios con una escala para caracterizar los niveles de ejecución a fin de juzgar la calidad de la tarea $o$ actividad realizada por los estudiantes. Tienen como propósito comprobar el nivel de dominio sobre los conceptos y las destrezas en determinadas tareas. Además ayudan a examinar fortalezas y debilidades en diversos tipos de aprendizaje. El procedimiento para la preparación de una rúbrica puede resumirse en los siguientes pasos:

च Seleccionar los objetivos (aprendizajes esperados) que fundamentan la tarea o actividad a realizar por parte del alumno.

$\square$ Identificar todos los posibles criterios que representan los comportamientos o ejecuciones esperados por los estudiantes al ejecutar la tarea.

$\square$ Organizar los criterios por niveles de efectividad.

च Asignar un valor numérico de acuerdo al nivel de ejecución.

Cada nivel debe tener descrito los comportamientos o ejecuciones esperados por los estudiantes. El estudiante debe conocer anticipadamente los criterios con los que será evaluado, siendo recomendable que éste se autoevalúe utilizando la rúbrica.

Particularmente existe una herramienta que podría facilitar la construcción de una rúbrica en [21], donde están disponibles plantillas para su construcción y rúbricas ya creadas según distintos temas y enfoques. Esta herramienta fue utilizada en este trabajo.

\section{APLICACIÓN}

Las anteriores consideraciones fueron utilizadas para la formulación de dos programas de asignaturas pertenecientes a la Facultad de Ingeniería de la Universidad del Bío-Bío.

Se presenta a continuación un breve resumen de su aplicación para la formulación de uno de los dos programas aludidos, el que corresponde a la asignatura de Accionamientos Eléctricos de la carrera de Ingeniería de Ejecución en Electricidad, luego de haber realizado el estudio del perfil del egresado y las áreas de dominio de las competencias profesionales declaradas por la institución. Se ha tomado en cuenta la identificación de las competencias derivadas del diagnóstico y plan formativo deseable, considerando el aporte de la asignatura tratada al plan de estudios y al perfil del egresado tal como se menciona en [22].

Despliegue de los aprendizajes esperados en las dimensiones: conceptual, procedimental y actitudinal

Los objetivos (aprendizajes esperados o resultados de aprendizajes esperados) al finalizar la asignatura por parte del alumno son separados en los distintos dominios que se ha mencionado. Estos fueron escogidos según el aporte de la asignatura al plan de estudios y al perfil del egresado.

\section{Aprendizaje cognitivo}

El alumno será capaz de:

$\square$ Identificar los tipos de accionamientos eléctricos en corriente alterna y corriente continua.

$\square$ Conocer las distintas normas asociadas al diseño y requerimientos de operación de los distintos componentes de un accionamiento eléctrico.

$\square$ Reconocer los diferentes componentes de un accionamiento eléctrico.

$\square$ Comprender el rol de cada componente dentro de un accionamiento eléctrico.

$\square$ Comparar las diferentes formas de control de velocidad de un motor eléctrico.

$\square$ Comprender la función de los parámetros más importantes de configuración de equipos utilizados en los accionamientos de motores eléctricos (Partidor suave, variador de frecuencia y chopper).

$\square$ Seleccionar un motor eléctrico para una aplicación dada.

$\square$ Recomendar los equipos eléctricos para el arranque y control de velocidad de un motor eléctrico.

$\square$ Evaluar el comportamiento de la carga sobre un accionamiento y sobre la red eléctrica.

\section{Aprendizaje procedimental}

El alumno será capaz de:

$\square$ Modelar cada uno de los componentes de un accionamiento eléctrico.

$\square$ Resolver problemas que involucren accionamientos eléctricos.

$\square$ Operar en forma básica equipos utilizados en accionamientos eléctricos (partidor suave, variador de frecuencia y chopper). 
■ Operar equipos para la visualización, medición y registro de variables eléctricas asociadas a la operación de un accionamiento eléctrico (voltaje, corriente, potencia, factor de potencia, contenido armónico y velocidad).

$\square$ Utilizar software para controlar en forma básica un accionamiento eléctrico (por ejemplo: SIMOVIS, variador de frecuencia Siemens).

$\square$ Utilizar software para analizar las variables eléctricas medidas o registradas, producto de la operación de un accionamiento eléctrico (DRANVIEW, POWERLOG).

\section{Aprendizaje actitudinal}

El alumno será capaz de:

च Valorizar la ventaja del trabajo cooperativo en la formación técnica.

$\square$ Valorizar los aspectos normativos de diseño asociados a cada componente dentro de un accionamiento eléctrico (motor eléctrico, partidor suave, variador de frecuencia y chopper).

च Valorizar los aspectos normativos de operación asociados a las perturbaciones al ambiente (Compatibilidad Electromagnética CEM) y la red eléctrica (Contaminación armónica) por parte de los equipos que componen un accionamiento eléctrico.

$\square$ Valorizar los protocolos de seguridad para la operación de equipos utilizados en el accionamiento de motores eléctricos.

Estos aprendizajes esperados definen los contenidos y la forma de realización de la asignatura. De éstos, es posible identificar cierto contenido base o clave para la generación del nuevo conocimiento que requiere la asignatura, particularmente: El Sistema Internacional de Unidades, clasificación de las cargas, características de los motores de corriente continua, características de los motores de corriente alterna, ecuaciones para el estudio dinámico del accionamiento y función de transferencia del motor de corriente continua.

\section{Identificación de las estrategias formativas}

Las estrategias formativas permiten emitir un juicio sobre el desempeño del estudiante con la finalidad de ayudarlo a aprobar el curso, éstas no se califican. La estrategia a utilizar es (i) la realización de un cuestionario y/o encuesta diagnóstico de forma de identificar las capacidades de entrada de los alumnos en las tres materias que convergen, según la malla de la carrera, en la asignatura de Accionamientos Eléctricos: Máquinas Eléctricas, Control Automático y Electrónica de Potencia. (ii) La retroalimentación luego de cada evaluación como test, certamen, tarea, informe de laboratorio y proyecto. $\mathrm{La}$ retroalimentación es hecha de dos maneras: (i) mediante el uso de pautas de cotejo en el dominio actitudinal con referencia a comportamientos y actitudes durante el desarrollo de los laboratorios y (ii) la reunión del grupo de trabajo o alumno con el profesor de manera de discutir los errores cometidos en los productos mencionados.

\section{Selección y organización de las actividades}

Las actividades en las que participará el alumno se pueden dividir en actividades formativas y de evaluación.

\section{Actividades formativas}

$\mathrm{Al}$ inicio de la asignatura se describe lo que se espera del alumno durante el curso, delineando su actuar, formato de presentación de proyectos, asistencia y descripción de la asignatura. Por otro lado se realizará:

च Un cuestionario o encuesta diagnóstico para medir la condición de entrada de los participantes de la asignatura.

$\square$ Actividades de retroalimentación. Al finalizar cada proceso de evaluación, particularmente para test, certamen, tareas y proyecto, se realizará una revisión de los mismos de forma de corregir terminología técnica, aplicación de conocimientos, análisis de resultados y conclusiones. Esta revisión es hecha en forma de mesa redonda para el caso de los proyectos al momento de la presentación oral y en forma individual o grupal según corresponda para el caso de las tareas, test y certámenes.

$\square$ Actividades de estudio de casos. En donde se fomentan las habilidades de toma de decisiones, resolver problemas, trabajar de forma colaborativa, desarrollo de la capacidad de análisis y pensamiento crítico (los casos corresponden a trabajos de seminario terminados en el área de accionamientos eléctricos).

$\square$ La técnica de One Minut Paper (actividad de cierre de clases), consistente en la realización de dos o tres preguntas abiertas al finalizar cada clase, de esta forma se fomentarán el análisis, 
la atención, la integración de información, la capacidad de escucha y de aprender por parte del alumno.

\section{Actividades de evaluación}

Las actividades de evaluación deben cubrir los tres dominios del saber del alumno (competencias).

$\square$ Los aspectos del saber serán evaluados mediante dos test y dos certámenes, que miden la adquisición de aspectos teóricos de la asignatura, por ejemplo, ítem verdadero-falso, completación de la oración, términos pareados y preguntas de respuesta abierta. Además, la capacidad de análisis, síntesis y aplicación serán evidenciados mediante la realización de tareas (de tipo investigativo y de resolución de problemas) y un proyecto de cierre de la asignatura, ambos evidenciados mediante el uso de rúbricas.

$\square$ Los aspectos del saber hacer serán medidos mediante pautas de cotejo para las actividades de laboratorio y rúbricas para los informes de laboratorio, tareas, informe de proyecto de cierre de asignatura y la presentación oral del mismo. Entre otros aspectos, el alumno debe usar la capacidad de búsqueda y selección de información, además de la lectura inteligente, capacidad de expresión y la organización de ideas.

$\square$ Los aspectos del saber ser serán evaluados por la observación utilizando pautas de cotejo para el dominio del saber ser en el laboratorio, considerando la colaboración, puntualidad, responsabilidad, pulcritud en su trabajo, trabajo en equipo, etc. Otros aspectos del saber ser serán evaluados por observación durante la presentación oral de los proyectos utilizando rúbricas. Las pautas de cotejo constituirán retroalimentación para el alumno al finalizar la asignatura.

\section{Asignación de créditos}

Actualmente la asignatura de Accionamientos Eléctricos tiene asignado cinco créditos. Sin embargo, estos créditos sólo consideran el trabajo presencial del alumno, ya sea en el aula o en el laboratorio. Por otro lado, la asignatura no contempla horas de práctica.

Ya que se ha modificado la perspectiva de la asignatura, se ha considerado 1 hora/semana de práctica de ejercicios, conservando las horas de clases teóricas de 4 horas/semana y de laboratorio de 2 horas/semana. Con esta distribución las horas de actividades presenciales del alumno son 7 horas/ semana. Las actividades no presenciales a considerar son aquellas relacionadas con:

$\square$ Actividades teóricas: realización de tareas, proyecto de cierre de la asignatura y de preparación para las evaluaciones tales como los certámenes y test, entre otros (1 hora/semana por cada hora teórica presencial).

च Actividades prácticas: Resolución de ejercicios. (2 horas/semana por cada hora de práctica presencial).

$\square$ Actividades de laboratorio: preparación de informe de laboratorio (2 horas/semana por cada hora de laboratorio presencial).

El total de horas en actividades no presenciales a considerar corresponde a 10 horas/semana, por lo tanto la carga académica del alumno es de 17 horas/ semana.

Si consideramos que la carrera de Ingeniería de Ejecución en Electricidad es una carrera de 4 años con 18 semanas semestrales (16 semanas académicas más dos semanas de exámenes o equivalentes) y que el tiempo promedio que destina el alumno para cumplir la carga académica del semestre es de 50 horas/semana a tiempo completo, se tiene que el alumno ocupa semestralmente 900 horas/semestre para cumplir con la carga académica. Este último valor es la cantidad de horas que debe repartir entre las distintas obligaciones con las asignaturas que esté cursando. Si se considera el primer principio para la asignación de créditos, según el Sistema de Créditos Transferibles chileno, tendremos la equivalencia de 60 créditos anuales con 1.800 horas/año ó 30 créditos semestrales con 900 horas/semestre (suponiendo la distribución de créditos anuales de manera equitativa en ambos semestres se verifica que un crédito SCT equivale a 30 horas/semestre para el caso de la Universidad). Por lo anterior, si la asignatura requiere de 17 horas/semana ó 306 horas/semestre de trabajo a tiempo completo, su cantidad de créditos en el SCT sería de 7. El cálculo anterior supone conocidas las horas de trabajo no presencial del alumno y que la hora pedagógica en la Universidad del Bío-Bío es de 40 minutos a diferencia de la cronológica de 60 minutos. Particularmente la Universidad ha desarrollado una herramienta que realiza el cálculo de 
los créditos SCT en forma automática considerando la información dada inicialmente en esta sección.

\section{CONCLUSIONES}

Este trabajo presenta las consideraciones más importantes en el ámbito conceptual y de aplicación de la enseñanza por competencias aplicadas a la formulación de un programa de asignaturas basado en esta metodología. Particularmente se muestra el proceso de formulación del programa de la asignatura de Accionamientos Eléctricos. El programa de asignaturas formulado constituye un ejemplo base para la futura implementación del modelo educativo de la Universidad del Bío-Bío en el Departamento de Ingeniería Eléctrica y Electrónica, el cual se encuentra en proceso del mismo. Este trabajo es uno de los primeros esfuerzos dentro del Departamento en apoyo a la implementación del modelo educativo que ha definido la Institución.

\section{AGRADECIMIENTOS}

Los autores agradecen a la Universidad del BíoBío por el apoyo prestado a este trabajo a través del proyecto FDD2008-09, complementado con el proyecto FDD2011-12, actualmente en curso.

\section{REFERENCIAS}

[1] Organización Internacional del Trabajo. Copyright (C) 1996-2011. Fecha de consulta: 5 de abril de 2011. URL: http://www.ilo.org/

[2] Centro Interamericano para el Desarrollo del Conocimiento en la Formación Profesional. Copyright (C) 1996-2011. Organización Internacional del Trabajo (OIT). Fecha de consulta: 5 de abril de 2011. URL: http://www.oitcinterfor.org/

[3] Organización Panamericana de la Salud. Copyright (C) 2010. Fecha de consulta: 5 de abril de 2011. URL: http://new.paho.org/

[4] J. Corrales y F. Salgado. "Innovación Didáctica hacia una Educación Centrada en el Estudiante en Laboratorio de Sistemas Eléctricos de Potencia”. Presentado a Ingeniare. Revista chilena de ingeniería para revisión por comité editorial. Abril, 2011.

[5] N. Chomsky. "Chomsky Info". Copyright (C) 2010. Fecha de consulta: 5 de abril de 2011. URL: http://www.chomsky.info
[6] Asociación Francesa de Normalización. Copyright (C) 2011. Fecha de consulta: 5 de abril de 2011. URL: http://www.afnor.org/

[7] Una Introducción a Tunning Educational Structures in Europe. La contribución de las universidades al proceso de Bolonia. Socrates-Tempus. Fecha de consulta: 5 de abril de 2011. URL: http://tuning.unideusto. org/tuningeu/images/stories/template/ General_Brochure_Spanish_version.pdf

[8] B.S. Bloom. "Taxonomy of educational objectives: The classification of educational goals”. Handbook I, Cognitive Domain. Longmans Green \& Co., New York. 1956.

[9] L.W. Anderson and D.R. Krathwohl. "A Taxonomy for Learning, Teaching, and Assessing: A revision of Bloom's Taxonomy of Educational Objectives". New York: Longman, 2001.

[10] B.S. Bloom, B.B. Masia and D.R. Krathwohl. "Taxonomy of Educational Objectives". Volume II: The Affective Domain. David McKay Company, Inc. New York. 1964.

[11] R.H. Dave. "Developing and Writing Behavioural Objectives". R. J. Armstrong Ed., Educational Innovators Press. Tucson. Arizona. 1970.

[12] W.R. Dawson. "Extensions to Bloom's Taxonomy of Educational Objectives". Putney Publishing. Sydney, Australia. 1998.

[13] E. Simpson. "The Classification of Educational Objectives in the Psychomotor Domain: The Psychomotor Domain”. Gryphon House. Vol. 3. Washington DC., USA. 1972.

[14] A. Harrow. "A Taxonomy of the Psychomotor Domain-A Guide for Develoing Behavioral Objectives". David McKay Company, Inc., New York. 1972.

[15] T. Ferris and S. Aziz. "A Psychomotor Skills Extension to Bloom's Taxonomy of Education Objectives for Engineering Education". Exploring Innovation in Education and Research, iCEER, Tainan, Taiwan. March, 1-5, 2005.

[16] F. Frabboni. "La scuola di base a tempo lungo". Ed. Liguore, Nápoles. 1984.

[17] J.S.B. Parraguez. "Designing Modules for Competence Based Curriculum". Conference on Electronic, Robotics and Automotive Mechanics, CERMA, pp. 763-768. September, 25-28, 2007. 
[18] CRUCH Consejo de Rectores de las Universidades Chilenas. "Guía Práctica para la Instalación de SCT-CHILE. Sistema de Créditos Académicos Transferibles". Fecha de consulta: 5 de abril de 2011. URL: http://www. sct-chile.cl/download/Guia_Practica_SCT.pdf

[19] Sistema de Créditos Académicos Transferibles. Copyright () 2007. Fecha de consulta: 5 de abril de 2011. URL: http://www.sct-chile.cl

[20] E. Himmel, M. Olivares y J. Zabalza. "Hacia una Evaluación Educativa. Aprender para evaluar y evaluar para aprender". Ministerio de Educación y Pontificia Universidad Católica de Chile. 1999.

[21] Rubistar. Copyright (C) 2000-2008. ALTEC University of Kansas. Fecha de consulta: 4 de abril de 2011. URL:

http://rubistar.4teachers.org

[22] F. Castro, G. Gómez y H. Lira. "Propuesta de modelo educativo Universidad del BíoBío". Documento base para la discusión. Diciembre 2006. 